\title{
Lanthanum oxide doping effect on dc electrical conductivity of ferroelectrics sodium vanadate and rubidium vanadate
}

\author{
D V PAWAR, T S MAGDUM and S H CHAVAN* \\ Department of Physics, Shivaji University, Kolhapur 416004, India \\ MS received 17 June 1994; revised 19 September 1994
}

\begin{abstract}
The temperature dependence of de electrical conductivity was measured by two-probe technique in the vicinity of phase transition point for ferroelectrics sodium vanadate and rubidium vanadate doped with different concentrations of $\mathrm{La}_{2} \mathrm{O}_{3}$. These materials show a sharp change in conductivity at their phase transition temperatures. The results were found to obey the conventional exponential law and the activation energies were calculated for ferroelectric and paraelectric states. It was found that activation energy in ferroelectric phase is smaller than in the paraelectric phase. The activation energy increases slowly with increase in doping concentration of $\mathrm{La}_{2} \mathrm{O}_{3}$ up to $0.1 \mathrm{~mol} \%$, however, it decreases with further increase in doping concentration, in both ferro and para states. The de electrical conductivity below the Curie temperature is of mixed type (ionic-electronic) while it is electronic type above the Curie temperature.
\end{abstract}

Keywords. Ferroelectrics; conductivity; phase transition; activation energy.

\section{Introduction}

Electrical conductivity is one of the important electrical characteristics of dielectric materials required not only for practical applications but also for the interpretation of various physical phenomena.

It was reported by Grawford (1959) that almost all ferroelectric materials which possess high Curie temperature lose their piezoelectric properties at temperatures considerably below their Curie points. Guruvich and Rez (1960) showed that the increase in electrical conductivity of these materials at high temperatures was due to loss of piezoelectric properties. The structural aspects of alkali metal vanadates were reported by Feigelson et al (1972) and they have shown that $\mathrm{NaVO}_{3}$ belongs to the monoclinic system with space group $\mathrm{Cc}$ at room temperature. The crystal chemistry of $\mathrm{M}^{+} \mathrm{VO}_{3}\left(\mathrm{M}^{+}=\mathrm{Li}, \mathrm{Na}, \mathrm{K}, \mathrm{NH}_{4}, \mathrm{Rb}, \mathrm{Cs}\right.$ and $\left.\mathrm{Tl}\right)$ pyroxenes were studied by Hawthorne and Calvo (1977) who concluded that $\mathrm{NaVO}_{3}$ is monoclinic with space group $\mathrm{Cc}$ while $\mathrm{RbVO}_{3}$ is orthorhombic with space group $\mathrm{Pbcm}$.

The electrical conductivity of the rare earth vanadate $\mathrm{LnVO}_{3}(\mathrm{Ln}=\mathrm{La}-\mathrm{Lu}$ and Y) compound was studied by Sakai et al (1976). Verma and Lal (1981) reported that the heavy rare-earth tungstates are mixed (ionic-electronic) conductors. The electrical transport in light and heavy rare earth vanadates was studied by Gaur and Lal (1985, 1986).

The aim of present communication is to study the dependence of dc electrical conductivity of sodium vanadate and rubidium vanadate doped with different concentrations $(0.025$ to $3 \mathrm{~mol} \%)$ of lanthanum oxide.

\footnotetext{
*For correspondence
} 


\section{Experimental}

The $\mathrm{NaVO}_{3}$ and $\mathrm{RbVO}_{3}$ ceramics used in this investigation were prepared from a stoichiometric mixture of $\mathrm{M}_{2}^{+} \mathrm{Co}_{3}\left(\mathrm{M}^{+}=\mathrm{Na}, \mathrm{Rb}\right)$ and vanadium pentoxide $\left(\mathrm{V}_{2} \mathrm{O}_{5}\right)$ by the usual ceramic technique following the method adopted by Feigelson et al (1972). These stoichiometric mixtures were slowly heated in a platinum crucible inside a globar furnace and fired at $750^{\circ} \mathrm{C}$ for $5 \mathrm{~h}$ and then cooled gradually to room temperature. The modified vanadates were prepared by taking different molar concentrations of $\mathrm{La}_{2} \mathrm{O}_{3}(99.9 \%$ purity) as an additive. Every batch was dry-mixed and then wet-mixed with ethyl alcohol in an agate mortar. After complete evaporation of alcohol, the batches were heated in a platinum crucible at $750^{\circ} \mathrm{C}$ for $5 \mathrm{~h}$ inside globar furnace and cooled to room temperature. The ceramic samples were then ground to pass 120 mesh sieve. Crystallinity was confirmed with X-ray diffraction.

Sample pellets were prepared in the form of disc of $1 \mathrm{~cm}$ diameter and about $0.2 \mathrm{~cm}$ thickness by applying $7.6 \times 10^{7} \mathrm{~kg} / \mathrm{m}^{2}$ pressure using a hydraulic press. The pellets were sintered on a platinum foil at $500^{\circ} \mathrm{C}$ for $4 \mathrm{~h}$. The two opposite faces of each pellet were polished and silvered with air-drying silver paste to achieve good electrical contact.

The experimental set-up consists of globar furnace, a digital dc microvoltmeter (VMV 15) with picoammeter adaptor, a transistorized power supply unit to provide dc electrical field, digital multimeter and a temperature controller. To ensure that contacts between electrodes and pellet interfaces were ohmic, the current density $(J)$-electric field $(E)$ characteristics for all samples at constant temperature were studied. The $J-E$ characteristics are linear up to the electric field of $200 \mathrm{~V} / \mathrm{cm}$. Above this critical field, the increase of $J$ with $E$ becomes non-linear. The linear plot below $200 \mathrm{~V} / \mathrm{cm}$ indicates the ohmic contact. In all measurements the electric field $(100 \mathrm{~V} / \mathrm{cm})$ was maintained below the critical field $(200 \mathrm{~V} / \mathrm{cm})$. The measurement of dc electrical conductivity was based on the dc resistivity data obtained by two-probe method. The pellet was slowly heated in a furnace by applying dc voltage of $100 \mathrm{~V} / \mathrm{cm}$ in series with pico-ammeter. The dc electrical conductivity was obtained from resistivity data in the vicinity of phase transition points. To ensure whether conductivity is ionic, electronic or mixed (ionic-electronic), the variation of dc electrical conductivity with time at a constant temperature was studied both in the ferroelectric and paraelectric states.

\section{Results and discussion}

The results of the measurement of dc electrical conductivity in pure and doped $\mathrm{NaVO}_{3}$ and $\mathrm{RbVO}_{3}$ obey the well known equation

$$
\sigma=\sigma_{0} \exp (\Delta E / k T),
$$

where $\Delta E$ is the activation energy, $\sigma_{0}$ a constant, $\sigma$ the electrical conductivity at temperature $T$ and $k$ the Boltzmann constant. The variation of $\log \sigma$ with $1 / T$ for pure $\mathrm{NaVO}_{3}$ and doped with different concentrations of $\mathrm{La}_{2} \mathrm{O}_{3}(0 \cdot 025,0 \cdot 05,0 \cdot 1$, $0.5 \mathrm{~m}, 1$ and $3 \mathrm{~mol} \%$ ) is shown in figure 1 , while for pure $\mathrm{RbVO}_{3}$ and doped $\mathrm{La}_{2} \mathrm{O}_{3}$ $(0.025,0.05,0.1,0.5,1$ and $3 \mathrm{~mol} \%)$ is shown in figure 2 . It is clear from figures 1 and 2 that the dc electrical conductivity increases exponentially with increasing temperature. 


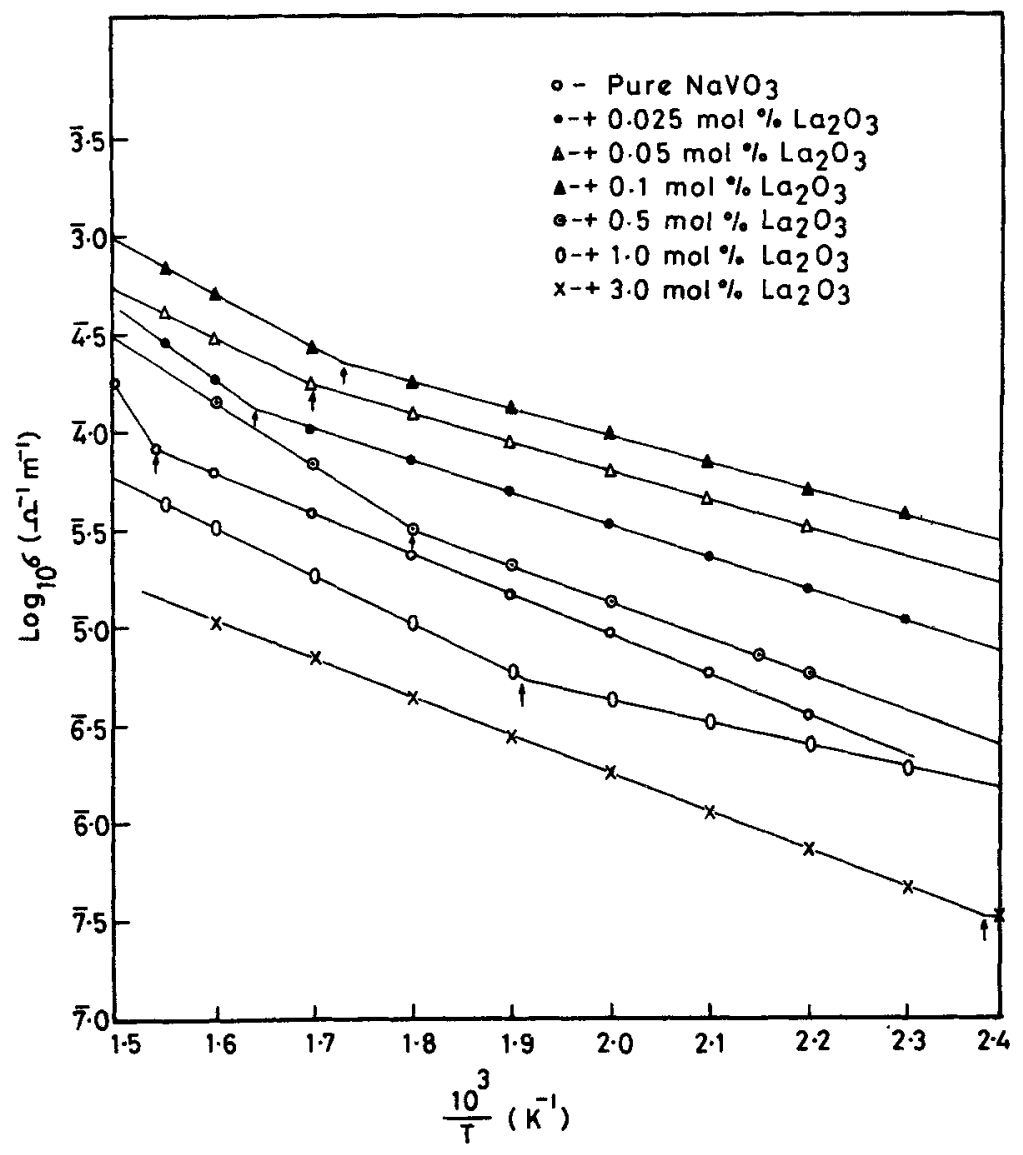

Figure 1. Variation of logarithmic conductivity (in $\Omega^{-1} \mathrm{~m}^{-1}$ ) with $1 / T$ for pure and doped $\mathrm{NaVO}_{3}$.

A sharp change in conductivity is observed at the transition temperature $\left(T_{\mathrm{c}}\right)$. These transition temperatures $\left(T_{\mathrm{c}}\right)$ are ferroelectric Curie temperatures of respective samples, $648 \mathrm{~K}$ for $\mathrm{NaVO}_{3}$ and $643 \mathrm{~K}$ for $\mathrm{RbVO}_{3}$ which we have confirmed by hysteresis loop method (Sawyer and Tower 1930) and dielectric constant measurements. These temperatures are in good agreement with those reported previously (Patil et al 1989, 1990). The doping of $\mathrm{La}_{2} \mathrm{O}_{3}$ to $\mathrm{NaVO}_{3}$ and $\mathrm{RbVO}_{3}$ shows noticeable shift in the Curie temperature to lower temperature (figures 1 and 2) and is in good agreement with the results obtained by Gopala Krishnan and Seshamma (1991).

Figures 1 and 2 reveal that the magnitude of conductivity increases with increase of dopant content at low $\mathrm{La}_{2} \mathrm{O}_{3}$ concentrations $(0.025,0.05,0.1 \mathrm{~mol} \%)$ and exhibits a maximum at $0.1 \mathrm{~mol} \%$ and then decreases for higher dopant concentrations from 0.5 to $3 \mathrm{~mol} \%$ of $\mathrm{La}_{2} \mathrm{O}_{3}$. The enhancement in dc electrical conductivity for addition of $0.1 \mathrm{~mol} \%$ of $\mathrm{La}_{2} \mathrm{O}_{3}$ in $\mathrm{NaVO}_{3}$ and $\mathrm{RbVO}_{3}$ ceramics is attributed to a rather more solid state interaction that takes place in the materials. This might be due to the increase of density with addition of $\mathrm{La}_{2} \mathrm{O}_{3}$ up to $0.1 \mathrm{~mol} \%$ (table 1). Doping with $\mathrm{La}^{3+}$ may keep the grain size small and thereby expedite densifications. It indicates 


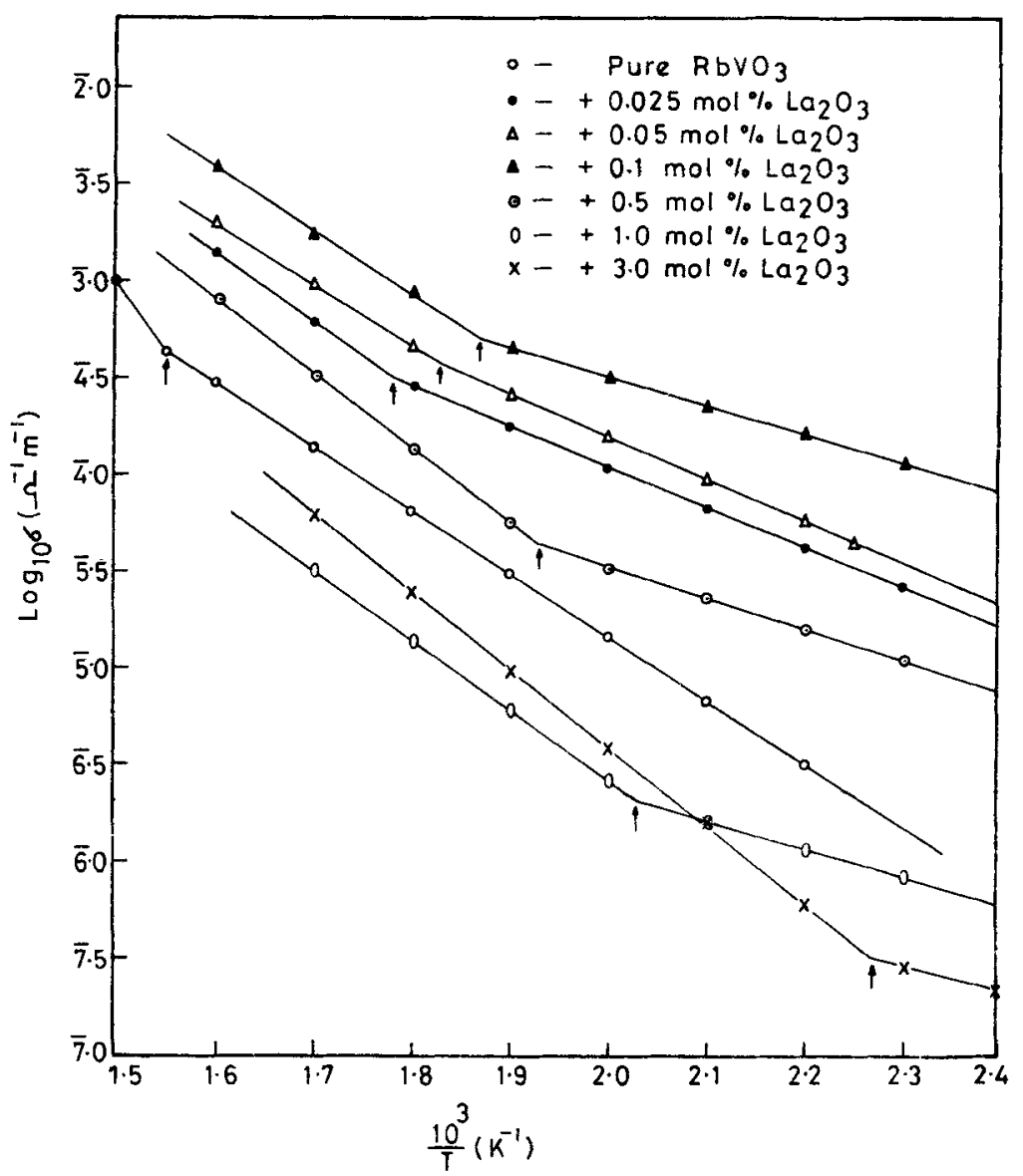

Figure 2. Variation of logarithmic conductivity (in $\Omega^{-1} \mathrm{~m}^{-1}$ ) with $1 / T$ for pure and doped $\mathrm{RbVO}_{3}$.

that the $0.1 \mathrm{~mol} \%$ doping may represent the solubility limit of $\mathrm{La}_{2} \mathrm{O}_{3}$ in $\mathrm{NaVO}_{3}$ as well as $\mathrm{RbVO}_{3}$ lattices. Similar results were reported by Weston et al (1969) for $\mathrm{Fe}_{2} \mathrm{O}_{3}$ doped PZT and by Tawfik et al (1978) for $\mathrm{Al}$ doped $\mathrm{BaTiO}_{3}$ ceramics. Thus increase in conductivity for doping up to $0.1 \mathrm{~mol} \%$ is because of the increasing concentration of cation vacancies in $\mathrm{NaVO}_{3}$ and $\mathrm{RbVO}_{3}$. Here $\mathrm{Na}^{+}$and $\mathrm{Rb}^{+}$ions are considered solely mobile ions on account of their low equivalent weights compared to $\mathrm{La}^{3+}$. The activation energies, both in ferroelectric and paraelectric states, and the experimental densities are summarized in table 1 for $\mathrm{NaVO}_{3}$ and $\mathrm{RbVO}_{3}$ ceramics doped with $\mathrm{La}_{2} \mathrm{O}_{3}$. Table 1 reveals that the activation energy in paraelectric state is higher than in the ferroelectric state.

The contribution to electrical conductivity is due to migration of ions or electrons or both. To investigate the nature of electrical conductivity, one has to distinguish the principal charge carriers. It is well known that, the dc electrical conductivity in a pure ionic conductor decreases with time and tends to zero after a very long time. In case of pure electronic conductor, the conductivity is independent of time, while in mixed (ionic--electronic) conduction, the dc electrical conductivity first decreases 
Table 1. Activation energies and densities of $\mathrm{NaVO}_{3}$ and $\mathrm{RbVO}_{3}$.

\begin{tabular}{llll}
\hline $\begin{array}{l}\mathrm{La}_{2} \mathrm{O}_{3} \\
\text { content } \\
(\mathrm{mol} \%)\end{array}$ & Activation energy $(\mathrm{eV})$ & $\begin{array}{c}\text { Ceramic density } \\
\left(\times 10^{3} \mathrm{~kg} / \mathrm{m}^{-3}\right)\end{array}$ \\
\cline { 2 - 4 } $\mathrm{NaVO}_{3}$ & Ferro & Para & \\
0.000 & 0.70 & 1.65 & 2.50 \\
0.025 & 0.33 & 0.79 & 2.59 \\
0.050 & 0.30 & 0.53 & 2.66 \\
0.100 & 0.29 & 0.60 & 2.72 \\
0.500 & 0.38 & 0.70 & 2.70 \\
1.000 & 0.25 & 0.50 & 2.67 \\
3.000 & 0.20 & 0.41 & 2.62 \\
$\mathrm{RbVO}_{3}$ & & & \\
0.000 & 0.63 & 1.48 & 3.08 \\
0.025 & 0.43 & 0.69 & 3.09 \\
0.050 & 0.42 & 0.62 & 3.16 \\
0.100 & 0.30 & 0.60 & 3.35 \\
0.500 & 0.31 & 0.72 & 3.23 \\
1.000 & 0.31 & 0.70 & 3.17 \\
3.000 & 0.27 & 0.77 & 3.10 \\
\hline
\end{tabular}

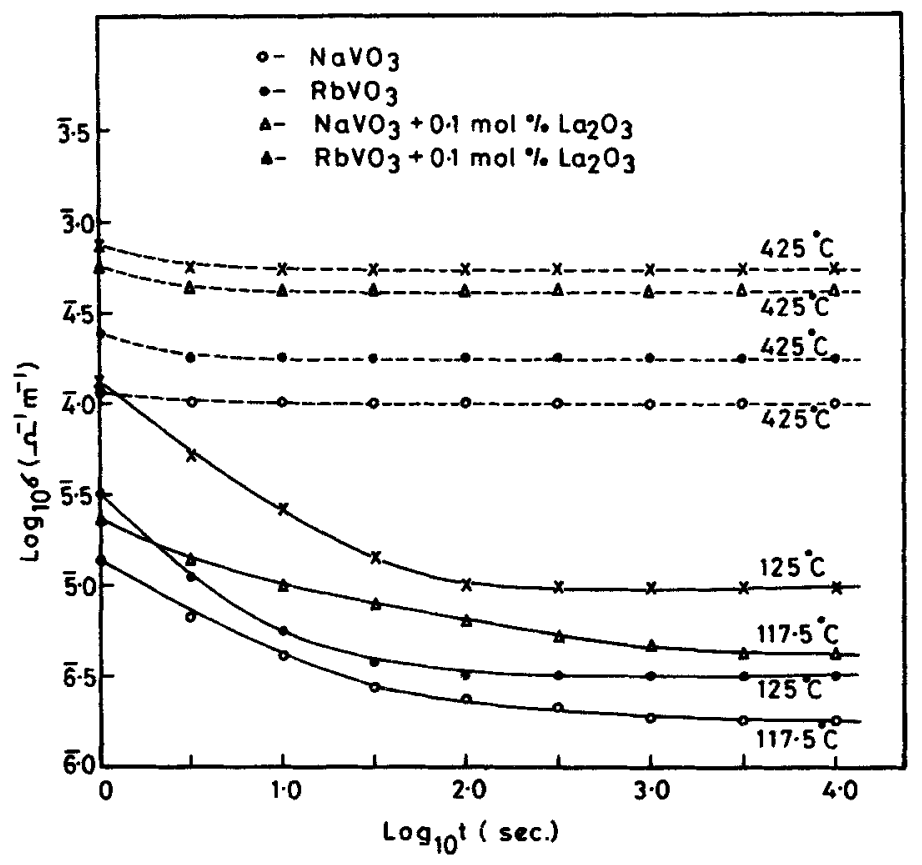

Figure 3. Variation of $\log \sigma$ with $\log t$ for $\mathrm{NaVO}_{3}, \mathrm{RbVO}_{3}, \mathrm{NaVO}_{3}+0 \cdot 1 \cdot \mathrm{mol} \% \mathrm{La}_{2} \mathrm{O}_{3}$ and $\mathrm{RbVO}_{3}+0.1 \mathrm{~mol} \% \mathrm{La}_{2} \mathrm{O}_{3}$. 
with time and then acquires a constant value after a long time. Figure 3 represents the variation of $\log \sigma$ with $\log t$ at a constant temperature, both in ferroelectric and paraelectric states, for pure $\mathrm{NaVO}_{3}$ and $\mathrm{RbVO}_{3}$ and doped with $0 \cdot 1 \mathrm{~mol}_{\%} / \mathrm{La}_{2} \mathrm{O}_{3}$.

Figure 3 reveals that the dc electrical conductivity below transition temperature is of mixed type (ionic-electronic) while above transition temperature it is electronic. Similar type of conductivity behaviour was reported by Chavan and Kulkarni (1993) and Rasal et al (1993).

\section{Conclusions}

(i) The dc electrical conductivity of all samples increases exponentially with increasing temperature, both in ferroelectric and paraelectric regions.

(ii) A sharp change in electrical conductivity is observed at phase transition temperature, indicating the ferroelectric Curie temperature of respective samples.

(iii) The addition of $\mathrm{La}_{2} \mathrm{O}_{3}$ to $\mathrm{NaVO}_{3}$ and $\mathrm{RbVO}_{3}$ shows noticeable shift in the Curie temperature to lower temperature.

(iv) The activation energies of all the samples are higher in the paraelectric region than in the ferroelectric region.

(v) The nature of electrical conductivity is of mixed type (ionic-electronic) in the ferroelectric region and electronic in the paraelectric region.

\section{References}

Chavan S H and Kulkarni A J 1993 J. Phys. Status Solidi a35 307

Feigelson R, Martin G and Johnson B J 1972 Crystal Growth (Germany) 13/14 686

Gaur K and Lal H B 1985 J. Mater. Sci. (Poland) 203167

Gaur K and Lal H B 1986 J. Mater. Sci. (USA) 212289

Gopala Krishnan R and Seshamma S 1991 Bull. Mater. Sci. 141349

Grawford A E $1959 \mathrm{Br}$. Commun. Electronics 6516

Guruvich V M and Rez I S 1960 Sov. Phys. Solid State 2624

Hawthorne F and Calvo C 1977 J. Solid State Chem. 22157

Patil V V, Kashid A P and Chavan S H 1989 Bull. Mater. Sci. 12133

Patil N B, Patil R T and Chavan S H 1990 Indian J. Pure Appl. Phys. 28276

Rasal S P, Magdum T S, Pawar D V and Chavan S H 1993 Ferroelectrics 13957

Sawyer C B and Tower C H 1930 Phys. Rev. 35269

Sakai T, Adachi G, Shiokawa J and Shinike T 1976 Mater. Res. Bull. 111295

Tawfik A, Sekina M M A and El-Nimr M K 1978 Indian J. Phys. A52 164

Verma B and Lal H 1981 Mater. Res. Bull. 161679

Weston T B, Webster A H and McNamara N H 1969 J. Am. Ceram. Soc. 52253 